\section{Kritik des ÄDA an den Präventionstagen der KBV}

D er Ärzteverband Deutscher Allergologen (ÄDA) vermißt eine sachgerechte Darstellung der Allergieprävention bei den diesjährigen Präventionstagen - einer Initiative des Deutschen Ärztebundes. Seine Kritik begründete der ÄDA auf dem 25 . Kongreß des Ärzteverbandes Deutscher Allergologen in Göttingen.

Es sei zwar einerseits zu begrüßen, daß das Thema „Berufsberatung bei allergischer Reaktion“ im Rahmen der Präventionstage aufgegriffen wird. Andererseits ist aber die allergologische Prävention mit all ihren notwendigen Aspekten - Allergieprävention bei Kindern, Gestaltung der Wohnver-

\section{Allergologie ' 98}

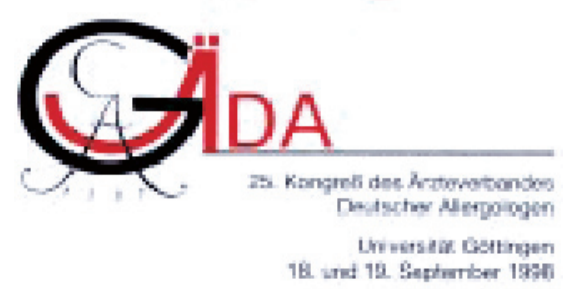

hältnisse, Berufsberatung, Arbeitsplatzgestaltung, Freizeitverhalten etc. - bisher in keiner Weise als kassenärztliche Leistung zugelassen. Und dies vor dem Hintergrund, daß inzwischen 24 bis $30 \%$ der deutschen Schulkinder an manifesten Allergien leiden. 12 Millionen Menschen leiden in der Bundesrepublik an Heuschnupfen, 4 Millionen an Asthma. Bis zu 80\% der aus gesundheitlichen Gründen abgebrochenen Berufsausbildungen sind auf eine Allergie zurückzuführen. Allergien sind die häufigste Ursache von Berufskrankheiten. Aufklärung und Prävention sind deshalb dringend notwendig, um ein weiteres Anwachsen der Erkrankungen zu verhindern.

Dr. med. Josef Wenning, Präsident des ÄDA: ,Unser Verband - der größte deutsche fachübergreifende Allergologenverband - hat sich langjährig und vielseitig für eine Aufnahme der Allergieprävention in die kassenärztli- che Versorgung eingesetzt. Leider wurden unsere Bemühungen bisher weder von der KBV noch von den Spitzenverbänden der Krankenkassen aufgegriffen. Wir bedauern daher die Tatsache, daß die KBV nicht die Zusammenarbeit mit allergologischen Verbänden gesucht hat und nun mit ihrer Themenwahl für die Präven-
D rof. Dr. Heinrich Worth, Chefarzt der I. Medizinischen Klinik des Klini-

kums Fürth ist neuer Vorsitzender der Deutschen Atemwegsliga e.V. Der bisherige stellvertretende Vorsitzende wurde damit Nachfolger von Prof. Dr. Ralf Wettengel, Bad Lippspringe.

Wettengel freute sich daß mit Worth eine Persönlichkeit die Führung der Atemwegsliga übernimmt, die bereits in den vergangenen Jahren die Arbeit der Liga in entscheidender Weise mit geprägt hat. Auf diese Weise ist sichergestellt, daß die Liga nach der Wahl darüber,
D. er Berufsverband Deutscher Baubiologen (VDB e.V.) wurde jetzt Ende August im Ökozentrum NRW gegründet. Ziel des ersten bundesweit aktiven Berufsverbandes ist es, die über zehnjährigen Erfahrungen der Baubiologie in Bezug auf sicheres und gesundes Wohnen fachkundig an ratsuchende Bürgerinnen und Bürger zu vermitteln. Bei Fragen zum gesunden Bauen, Wohnen und Einrichten oder zu Umweltrisiken in den eigenen vier tionstage nach außen hin einen falschen Eindruck zu erwecken versucht. Dabei hat sie sich bisher gegen jede Form der Allergieprävention gesperrt. Wir fordern heute erneut eine sofortige Aufnahme der Allergieprävention in das Spektrum kassenärztlicher Leistungen. Wir sind in jeder Weise zu einer sachgerechten Mitarbeit bereit."

Die Allergieprävention sollte unter Beteiligung der Fachverbände das zentrale Thema der Präventionstage 1999 sein, forderte Wenning.

\title{
Atemwegsliga mit neuem Vorstand
}

auch in Zukunft verantwortungsbewußt an der Umsetzung ihrer Aufgaben und Ziele arbeitet.

Neuer stellvertretender Vorsitzender ist Prof. D. Nolte aus Bad Reichenhall, der ebenfalls seit vielen Jahren aktiv im Ligavorstand mitarbeitet. Prof. Criée aus Göttingen ist weiterhin Schatzmeister. Daß nicht alles beim Alten bleibt, sondern auch frischer Wind weht, dafür wird Priv.-Doz. Dr. Rabe aus Großhansdorf sorgen. Rabe, der in Kürze den Lehrstuhl für Pneumologie in Leiden übernimmt, wurde als neues Mitglied in den Vorstand kooptiert.

\section{Gebührenfreies „,Gesund-Wohnen-Telefon“"}

Wänden können sich Hilfesuchende an die kostenlose Rufnummer (08 00) 2001007 von Montag bis Freitag zwischen 9 und 18 Uhr wenden. Vor allem bei Problemen mit Schimmelpilzen, Formaldehyd- oder Holzschutzmittelbelastungen sind Baubiologen kompetente Ansprechpartner. Weitere Informationen sind erhältlich bei der Bundesgeschäftsstelle des VDB, Oberwiesenthaler Straße 18, D91207 Lauf, Tel.: $09123 / 984012$ und der Faxnummer 091 23/9840 13. 